\title{
LARGE RATE OF NORMAL INJECTION NEAR TRAILING EDGE*
}

\author{
BY
}

\author{
L. TING AND A. ISMAIL
}

New York University, Bronx

1. Introduction. The problem of large surface blowing has attracted considerable attention recently ([1]-[8]). The problem arises when the rate of ablation or injection required for surface cooling is so large that the normal velocity at the surface is larger than the value allowed for in the boundary layer theory. The problem also arises when jets or surface injections are employed as control or lift devices. Simple models have been introduced to simulate the flow fields with massive ablation in porous injection ([1]-[6]). In those models two boundary conditions along the body surface are imposed; they are the prescribed normal velocity and the nonslip condition. Analyses have been carried out under the framework of the boundary layer theory in [1] and [2] and as an inviscid limit of the boundary layer theory in [3]. In [4], [5] and [6], the inner stream of the ablater or the injectant is assumed to be inviscid (but rotational to take care of the two boundary conditions) and special similar solutions are obtained for wedges or cones with a power law normal velocity prescribed along the wall. The usefulness of these solutions are hinged on the validity of the assumption that the influences of the upstream variations or singularities will die out very fast and that the feedback of the downstream variations are negligible. These assumptions have been taken for granted in many boundary layer analyses. 'The question of how quickly the upstream variations will die out has been examined in [9] and [10] and the references therein. For the analysis of the feedback due to downstream separation or shock boundary layer interaction [11], there is a strong doubt of the usefulness of boundary layer equations.

In order to be able to obtain solutions which take into account the upstream and downstream influences a simple model of flow field is analyzed. It simulates the flow field of injection with density $\rho_{i}$ from a reservoir of constant stagnation pressure $p_{s i}$ through slots normal to a surface moving at supersonic speed. For the analysis, the flow field of injectant is assumed to be inviscid and incompressible and to be uncoupled from the outer flow field by imposing a unique $p-\theta$ relationship between pressure and local inclination along the dividing streamline. These assumptions are justified by the restrictions on the difference between the stagnation pressure of the injectant and the pressure of the external stream $p_{\infty}$ so that (i) the pressure difference across the layer of injectant is larger than that allowed for by the boundary layer theory, (ii) the velocity of the injectant is much less than the speed of sound, $a_{i}$, of the injectant and (iii) the outer stream remains supersonic so that the $p-\theta$ relationship along the dividing stream-

* Received July 21, 1968; revised version received July 17, 1969. Work supported by Army Research Office, Durham, N. C., Contract No. DA-31-124-ARO-D-464. 
lines can be given by the linearized theory, the second order theory [12] or the hypersonic theory [13], whichever is appropriate. The corresponding restrictions on $p_{\bullet i}-p_{\infty}$ are

$$
\begin{aligned}
& \left(p_{s i}-p_{\infty}\right) /\left(\rho_{\infty}-q_{\infty}^{2}\right) \gg 1 / \operatorname{Re} \\
& \left(p_{s i}-p_{\infty}\right) /\left(\frac{1}{2} \rho_{j} a_{i}^{2}\right) \ll 1
\end{aligned}
$$

and

$$
p_{s i}-p_{\infty}<p^{*}-p_{\infty}
$$

where $\rho_{\infty}$ and $q_{\infty}$ are the density and the velocity of the external supersonic stream, $p^{*}-p_{\infty}$ is the pressure jump across a shock from Mach number $M_{\infty}$ to unity and Re is the Reynolds number with respect to the length $L$ of the injection region along the surface. For injection along a curved wall $\mathrm{Re}$ in $\mathrm{Eq}$. (1) should be replaced by $\mathrm{Re}^{1 / 2}$ and the radius of the curvature of the wall should be used as the reference length. Since the injectant has the same stagnation pressure, the flow field is irrotational and is a potential flow.

When the injection region is far ahead of the trailing edge, the potential flow field is partially bounded by the body surface and the dividing streamline emanating from the leading edge of injection region as shown in Fig. 1. Along the body surface the direction of flow, $\theta$, is prescribed. It is normal to the surface in the slotted region and tangential to the surface in the region without injection. Far downstream, the flow becomes uniform, i.e.,

$$
\theta \rightarrow \theta_{e}, \quad p_{i} \rightarrow p_{\infty}
$$

and

$$
q_{i} \rightarrow q_{j \infty}=\left[2\left(p_{s i}-p_{\infty}\right) / \rho_{i}\right]^{1 / 2} \quad \text { as } s \rightarrow \infty
$$

where $s$ is the arc length along the body surface. This is the model analyzed in [7], [8]. In [7], the body surface is a wedge and along the dividing streamline the condition of Ecq. (2) is replaced by a more restrictive one, $\left(p_{s i}-p_{\infty}\right) /\left(\rho_{\infty} q_{\infty}^{2}\right)=\epsilon \ll 1$, so that the linearized supersonic theory can be applied to relate pressure to local inclination, and analytical results have been obtained. The analytical results are confirmed by the numerical analysis of [8] by a successive iteration of the shape of the dividing streamline and a finite difference solution of the inner region. The numerical method has been

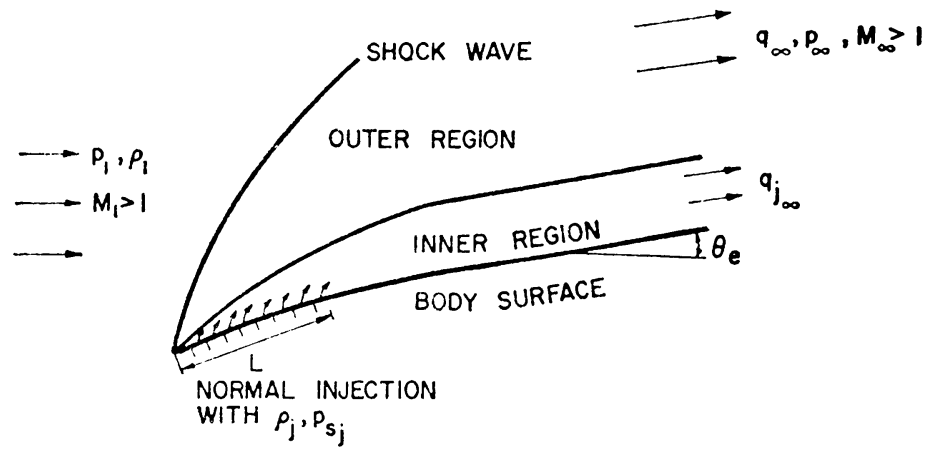

Fig. 1. Fluw field aroind an open body with large normal injection 


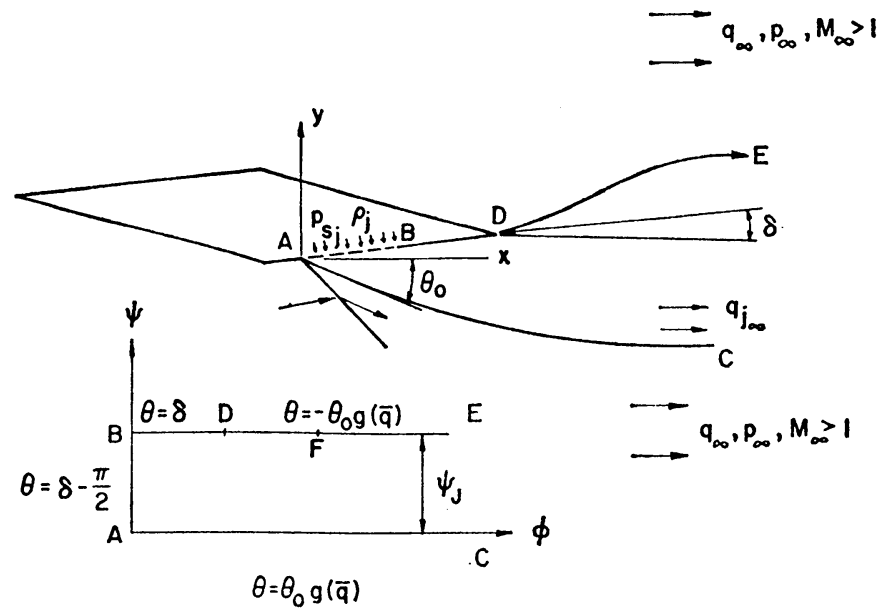

FIG. 2. Flow field for injection near trailing edge without separation region

employed in [8] to handle a more complicated boundary condition along the dividing stream, namely the Busemann second-order theory [12] or the hypersonic theory [13] and to handle a more general body shape, say a wedge with an ogive nose, and also to the axially symmetric problem.

When the injection region is not too far ahead of the trailing edge, as compared to the length of the slotted region, it seems to be logical to extend this model of flow field without separation by the addition of another dividing streamline from the trailing edge. This model of flow field and the boundary conditions for the potential flow of the injectant are shown in Fig. 2. A proof for the nonexistence of a potential solution for the proposed model is given in [14] when the inclination of the surface $\delta$ is less than the angle of deflection $\theta_{0}$ which will raise the pressure of the supersonic stream to the stagnation of its injectant $p_{s i}$, i.e.,

$$
-\theta_{0}<\delta<\theta_{0} .
$$

The inequality, $-\theta_{0}<\delta$, is necessary to insure that the injection on the lower surface will take place. The other one, $\delta<\theta_{0}$, is the extra restriction required for the mathematical proof of nonexistence of the potential solution. Without the extra restriction, this model of flow field without a finite separation region can be ruled out by the inclusion of the viscous effect as discussed in the last section.

For injection near trailing edge it is necessary to admit a separation region. An analytical solution for a modified model of the inviscid flow field including a constantpressure wake region is presented in the next section.

It should be pointed out here that the potential solutions are applicable to the slotted injection problems subjected to the restrictions of Eqs. (1), (2), and (3). They are not applinable to the massive porous injection or massive ablation problems for which the stagnation pressure is not a constant and the flow is rotational. Nevertheless, the importance of the upstream and dowistream conditions, in particular the presence of a nearby trailing edge as illustrated in the present slot injection problems, will also be valid for the massive porous injection or ablation problems.

2. Flow field with a separation region. A simple model for the flow field is shown 

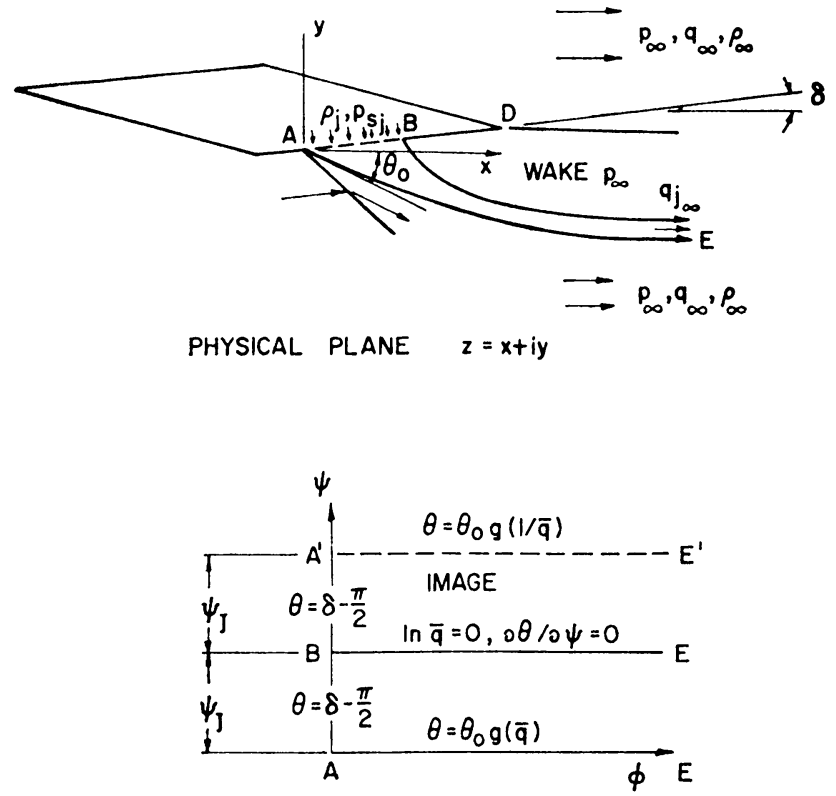

COMPLEX POTENTIAL PLANE $X=\phi+i \psi$

FIG. 3. Flow field for injection near trailing edge with separation region

in Fig. 3. There are three regions. The inner region of the injectant is separated from the outer supersonic flow field by the lower dividing streamline $A E$ and is separated from the wake region by the upper dividing streamline $B E . A B$ is the slotted segment on the airfoil. The constant-pressure wake region is separated from the upper supersonic flow field by a horizontal streamline from the trailing edge $D$ to downstream infinity. The pressure in the wake region and on the dividing streamline $B E$ is therefore $p_{\infty}$. For the potential flow of the injectant, the boundary conditions are

$$
\begin{aligned}
\ln \bar{q} & =\ln \left(q_{i} / q_{j \infty}\right)=0 & & \text { along } B E, \psi=\psi_{i}, \varphi>0 \\
\theta & =\delta-\pi / 2 & & \text { along } A B, 0<\psi<\psi_{i}, \varphi=0 \\
\theta & =\theta_{0} g(\bar{q}) & & \text { along } A E, \psi=0, \varphi>0
\end{aligned}
$$

and

$$
\theta \rightarrow 0, \ln \bar{q} \rightarrow 0 \quad \text { as } \varphi \rightarrow \infty .
$$

$\bar{q}$ is the nondimensional velocity and $\theta$ is the inclination. $\psi$ and $\varphi$ are the potential and stream functions and the total mass flux of the injectant, $\psi_{i}$, is a constant to be defined later. $g(\bar{q})$ will depend on the pressure and inclination relationship to be used for the outer superionic stream. $q_{j \infty \infty}$ is the velocity of the injectant at pressure $p_{\infty}$ and is related to the stagnation pressure by the Bernoulli equation $q_{j \infty}=\left[\left(p_{s i}-p_{\infty}\right) / 2\right]^{1 / 2} \cdot \theta_{0}$ is the angle of deflection which raises the pressure of the outer strean to $p_{s i}$. Eq. (6a) follows from the definition of $q_{\infty}$ and $g(0)=-1$ is due to the definition of $\theta_{0}$. Also shown in Fig. 3 are the complex potential plane $\chi(=\varphi+i \psi)$ and the corresponding boundary conditions. The logarithm of the complex conjugate velocity $\ln \bar{q}-i \theta$ denoted by 
$Q$ is an analytic function of the complex potential $\chi[15]$. With the aid of Cauchy Riemann equations, the boundary condition along $B E$, Eq. (6a), can be replaced by

$$
\partial \theta / \partial \psi=0 \quad \text { along } B E, \psi=\psi_{J}, \varphi>0
$$

By the method of reflection, the half strip $A B E A$ in $\chi$-plane can be extended to $A B A^{\prime} E A$ with the following boundary conditions:

$$
\begin{aligned}
& \theta=\theta_{0} g\left(\bar{q}^{-1}\right) \quad \text { along } A^{\prime} E^{\prime}, \psi=2 \psi_{J}, \varphi>0 \\
& \theta=\delta-\pi / 2 \text { along } A A^{\prime}, 0<\psi<2 \psi_{J}, \varphi=0 \\
& \theta=\theta_{0}(\bar{q}) \quad \text { along } A E, \psi=0, \varphi>0 \\
& Q \rightarrow 0 \quad \text { as } \varphi \rightarrow \infty \text {. }
\end{aligned}
$$

Boundary condition (8a) is obtained by noting that when $\theta$ is an even function of $\psi-\psi_{J}$, $\ln \bar{q}$ is an odd function, and therefore $1 / \bar{q}$ along $\psi=2 \psi_{j}$ is equal to $\bar{q}$ along $\psi=0$.

This half strip in the $\chi$-plane can be mapped into the upper $\zeta$-plane as shown in Fig. 4 by the transformation

$$
\zeta=\xi+i \eta=\cosh \left[\pi \chi /\left(2 \psi_{s}\right)\right] .
$$

The boundary conditions of Eqs. (8a, b, c, d) become

$$
\begin{array}{ll}
\theta=\theta_{0} g\left(\bar{q}^{-1}\right) & \text { for } \xi<-1, \eta=0 \\
\theta=\delta-\pi / 2 & \text { for } \quad|\xi|<1, \eta=0 \\
\theta=\theta_{0} g(\bar{q}) & \text { for } \xi>1, \eta=0
\end{array}
$$

and

$$
Q \rightarrow 0 \quad \text { as }|\xi| \rightarrow \infty .
$$

By means of the Cauchy integral formula [16] the solution $Q(\zeta)$ can be written as

$$
\begin{aligned}
Q(\zeta)=\ln \bar{q}-i \theta= & -1 / \pi \int_{-\infty}^{+\infty} \frac{\theta\left(\xi^{\prime}, 0\right) d \xi^{\prime}}{\xi^{\prime}-\zeta} \\
= & \left(\frac{1}{2}-\frac{\delta}{\pi}\right) \ln \frac{\zeta-1}{\zeta+1}-\frac{\theta_{0}}{\pi}\left[\int_{-\infty}^{-1} \frac{g\left[1 / \bar{q}\left(\xi^{\prime}, 0\right)\right] d \xi^{\prime}}{\xi^{\prime}-\zeta}\right. \\
& \left.+\int_{1}^{\infty} \frac{g\left[\bar{q}\left(\xi^{\prime}, 0\right)\right] d \xi^{\prime}}{\xi^{\prime}-\zeta}\right] .
\end{aligned}
$$

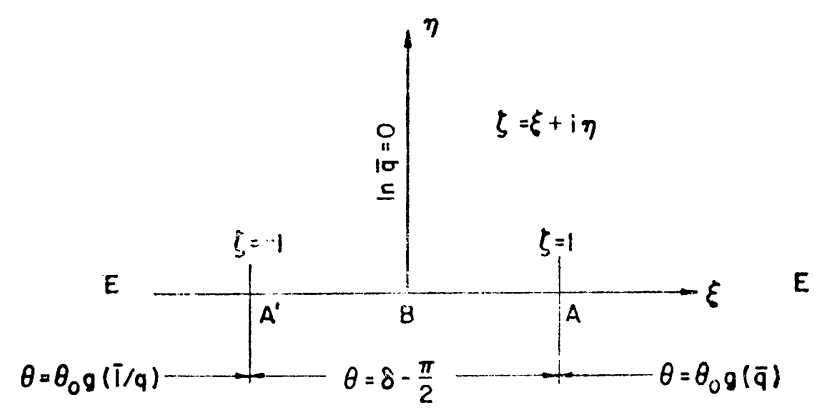

Fia. 4. The $x$-plane 
The argument of $\ln \{(\zeta-1) /(\zeta+1)\}$ is chosen to be zero along the real $\xi$ axis with $\xi>1$. Eq. (11) is a nonlinear integral equation similar to the one in [7] with the integral from $-\infty$ to -1 as the extra term. Since the function $g$ is bounded and vanishes as $\bar{q} \rightarrow 1$ when $|\zeta| \rightarrow \infty$, the integral equation will be solved by the method of successive iteration for sufficiently small $\theta_{0} / \pi$ in the same manner as [7].

$$
\begin{array}{r}
Q^{(n)}(\zeta)=\ln \bar{q}^{(n)}-i \theta^{(n)}=\left(\frac{1}{2}-\frac{\delta}{\pi}\right) \ln \frac{\zeta-1}{\zeta+1}-\frac{\theta_{n}}{\pi}\left[\int_{-\infty}^{-1} \frac{g\left[1 / \bar{q}^{(n-1)}\left(\xi^{\prime}, 0\right)\right]}{\xi^{\prime}-\zeta} d \xi^{\prime}\right. \\
\left.+\int_{1}^{\infty} \frac{g\left[\bar{q}^{(n-1)}\left(\xi^{\prime}, 0\right)\right]}{\xi^{\prime}-\zeta} d \xi^{\prime}\right] \quad n=2,3, \cdots
\end{array}
$$

The first two iteration solutions are

$$
Q^{(0)}(\zeta)=\left(\frac{1}{2}-\delta / \pi\right) \ln [(\zeta-1) /(\zeta+1)]
$$

with

$$
\ln \bar{q}^{(0)}=\left(\frac{1}{2}-\delta / \pi\right) \ln |(\zeta-1) /(\zeta+1)|
$$

and

$$
\begin{aligned}
Q^{(1)}(\zeta) & =\left(\frac{1}{2}-\frac{\delta}{\pi}\right) \ln \frac{\zeta-1}{\zeta+1}-\frac{\theta_{0}}{\pi}\left[\int_{-\infty}^{-1} \frac{g\left[1 / \bar{q}^{(0)}\right] d \xi^{\prime}}{\xi^{\prime}-\zeta}+\int_{1}^{\infty} \frac{g\left(\bar{q}^{(0)}\right) d \xi^{\prime}}{\xi^{\prime}-\zeta}\right] \\
& =\left(\frac{1}{2}-\frac{\delta}{\pi}\right) \ln \frac{\zeta-1}{\zeta+1}-\frac{\theta_{0}}{\pi} \int_{1}^{\infty}\left[\frac{1}{\xi^{\prime}-\zeta}-\frac{1}{\xi^{\prime}+\zeta}\right] g\left[\left(\frac{\xi^{\prime}-1}{\xi^{\prime}+1}\right)^{1 / 2-\delta / \pi}\right] d \xi^{\prime}
\end{aligned}
$$

The relation for the physical coordinates $x+i y$ having origin at point $A$ is given by the integral [16]

$$
\begin{aligned}
z^{(n)}=\int_{0}^{x} d \chi /\left(q_{j} e^{-i \theta}\right) & =\int_{0}^{x} \exp \left[-Q^{(n)}\right] d \chi / q_{i \infty} \\
& =\left[2 \psi_{J}^{(n)} /\left(\pi q_{i \infty}\right)\right] \int_{1}^{\zeta} \exp \left[-Q^{(n)}\right]\left(\zeta^{2}-1\right)^{-1 / 2} d \zeta
\end{aligned}
$$

for $n=1,2,3 \ldots$ The square root of $\zeta^{2}-1$ is positive along the real $\xi$ axis with $\xi>1$. It should be noted that $q^{(0)} \sim(\zeta-1)^{1 / 2-\delta / \pi}$ as $\zeta \rightarrow 1$ and the above integral has no meaning for $n=0$ when $\delta \leq 0$. This is due to the wrong singularity of $Q^{(0)}$ at $\zeta=1$ for which the lower dividing streamline inclined at angle $\theta \leq 0$ cuts into the wing. Since $Q^{(n-1)}=0$ at $\zeta=1$ and $g(0)=-1$, it can be shown that $Q^{(n)}$, for $n \geq 1$, possesses the correct singularity at $\zeta=1$, i.c., turning from $\theta=\delta-\pi / 2$ to $-\theta_{0} \cdot q^{(n)}$ for $n \geq 1$ behaves as $(\zeta-1)^{1 / 2-\left(\delta+\theta_{0}\right) / \pi}$ as $\zeta \rightarrow 1$. With $\theta_{0}>-\delta$, the lower dividing streamline inclined at angle $\theta=\theta_{0}$ lies below the wing surface and the above integral is finite for finite upper limit.

The constant $\psi_{J}^{(n)}$ is related to the length $A B$ by the condition

$$
\begin{aligned}
L e^{i \delta} & =\int_{0}^{\psi J} i d \psi /\left[q_{i} e^{-i(\delta-\pi / 2)}\right] \\
\text { or } L & =\left[2 \psi_{J}^{(n)} /\left(\pi q_{i \infty}\right)\right] \int_{0}^{1} d \xi\left|\exp \left(-Q^{(n)}\right)\right|_{\eta=0}\left(1-\xi^{2}\right)^{-1 / 2} .
\end{aligned}
$$


The parametric equations for the upper and the lower dividing streamlines are given by the integral of Eq. (15) with $-\infty<\zeta=\xi<-1$ and $\infty>\zeta=\xi>+1$ respectively.

Once the appropriate pressure-inclination relationship for the outer stream is chosen, these integrals can be evaluated by numerical integration.

When the linearized supersonic theory is applicable, i.e., $\left(p_{s j}-p_{\infty}\right) /\left(p_{\infty} q_{\infty}^{2}\right)=\epsilon \ll 1$, it follows that $\theta_{0} / \pi=\left(M_{\infty}^{2}-1\right)^{1 / 2} \epsilon / \pi \ll 1$, and the second iteration solution $Q^{(1)}$ is adequate for the description of the flow field including the transformation to the physical coordinates. The function $g(\bar{q})$ in this case is $-\left(1-\bar{q}^{2}\right)$ and Eq. (21) becomes

$$
\begin{aligned}
Q^{(1)}(\zeta) & =\left(\frac{1}{2}-\frac{\delta}{\pi}\right) \ln \frac{\zeta-1}{\zeta+1} \\
& +\frac{\theta_{0}}{\pi} \int_{1}^{\infty} d \xi\left[\frac{1}{\xi^{\prime}-\zeta}-\frac{1}{\xi^{\prime}+\zeta}\right]\left[1-\left(\frac{\xi^{\prime}-1}{\xi^{\prime}+1}\right)^{1-(2 \delta) / \pi}\right]
\end{aligned}
$$

To be consistent with the linearized supersonic theory $\delta / \pi$ should be of the order of $\theta_{0} / \pi$ or $\epsilon$; then the following approximate analytic solution can be obtained:

$$
\begin{aligned}
Q^{(1)}(\zeta) & =\left(\frac{1}{2}-\frac{\delta}{\pi}\right) \ln \frac{\zeta-1}{\zeta+1} \\
& -\frac{\theta_{0}}{\pi}\left[\frac{2}{1+\zeta}\left(\ln \frac{\zeta-1}{2}-\pi i\right)-\frac{2}{1-\zeta} \ln \frac{1+\zeta}{2}\right]+O\left(\epsilon^{2}\right) .
\end{aligned}
$$

The arguments of the logarithm inside the square brackets are restricted between $\pi$ and $-\pi$.

It should be pointed out here that although the correction $\left[Q^{(1)}-Q^{(0)}\right] / Q^{(0)}$ is of the order $\epsilon$ including the point $\zeta=1$, the correction in velocity $\left[\bar{q}^{(1)}-\bar{q}^{(0)}\right] / \bar{q}^{(0)}$ near $\zeta=1$ is not. If $Q^{(0)}$ is modified to have the same behavior near $\zeta=1$ as $Q^{(1)}$, a composite solution can be created

$$
\widetilde{Q}^{(0)}=\left(\frac{1}{2}-\frac{\delta}{\pi}-\frac{\theta_{0}}{\pi}\right) \ln \frac{\zeta-1}{\zeta+1}
$$

and the corresponding magnitude of the velocity is

$$
\tilde{q}^{(0)}=|(\zeta-1) /(\zeta+1)|^{1 / 2-\left(\delta+\theta_{0}\right) / x} .
$$

Not only will $\widetilde{Q}^{(0)}$ differ from $Q^{(1)}$ by an order of $\epsilon$, but $\tilde{q}^{(0)}$ will differ from $q^{(1)}$ by an order of $\epsilon$ including the stagnation point $\zeta=1$. The composite solutions can now be used for the transformation to the physical plane to yield the leading term of the solution.

Fig. 5 shows the upper and the lower dividing streamlines of the flow field of the injectant with $M_{\infty}=2, \delta=0$ and $\epsilon=0.1$. The pressure distribution is related to the magnitude of velocity by the Bernoulli equation, i.e.,

$$
\begin{aligned}
\left(p-p_{\infty}\right) /\left(p_{s i}-p_{\infty}\right) & =1-\bar{q}^{2} \\
& =1-\left[\dot{q}^{(0)}\right]^{2}+O(\epsilon) \\
& =1-|(\zeta-1) /(\zeta+1)|^{1-2\left(\theta_{0} \cdot \delta\right) / \tau}+O(\epsilon) .
\end{aligned}
$$

On the surface of the wing, $p$ is equal to $p_{\infty}$ behind the slotted region due to the assumption of a constant-pressure wake region. Along the slotted region $0<\zeta=\xi<1$ the 


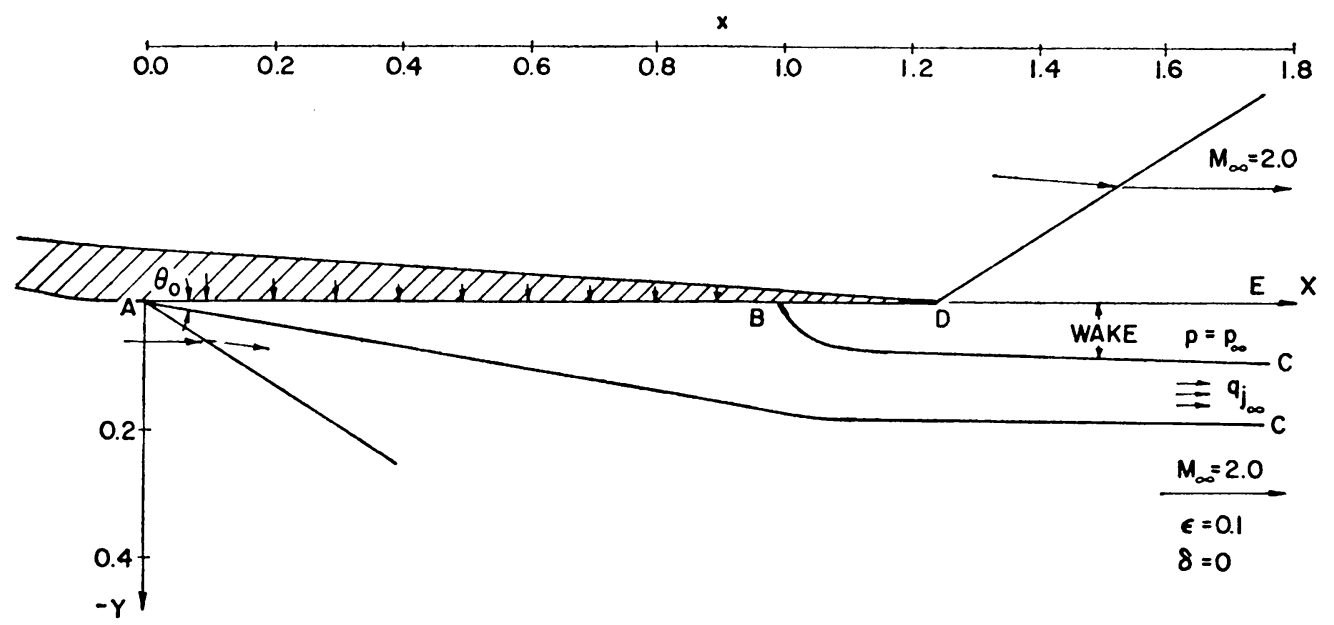

FIG. 5. Flow field with constant pressure wake $\left(M_{\infty}=2, \delta=0, \epsilon=0.1\right)$

arc length $s$ is related to the variable $\xi$ by means of Eqs. (15) and (16); i.e.,

$s / L=\int_{0}^{\xi} \frac{d \xi^{\prime}}{\bar{q}\left(\xi^{\prime}, 0\right)\left(1-\xi^{\prime 2}\right)^{1 / 2}} / \int_{0}^{1} \frac{d \xi^{\prime}}{\bar{q}\left(\xi^{\prime}, 0\right)\left(1-\xi^{\prime 2}\right)^{1 / 2}}=(1-\xi)^{\left(\theta_{0}+\delta\right) / \pi}+O(\epsilon)$.

Eqs. (21) and (22) provide pressure distribution along the surface of the wing in terms of the parameter $\xi$. Fig. 6 shows the pressure distribution along the surface for various values of $\left(\theta_{0}+\delta\right) / \pi$ where $\theta_{0}+\delta$ is the angle between the lower dividing streamline and the wing surface.

3. Discussion. In the study of normal injection of an incompressible fluid with constant stagnation pressure through a wing surface moving at high speed, the flow

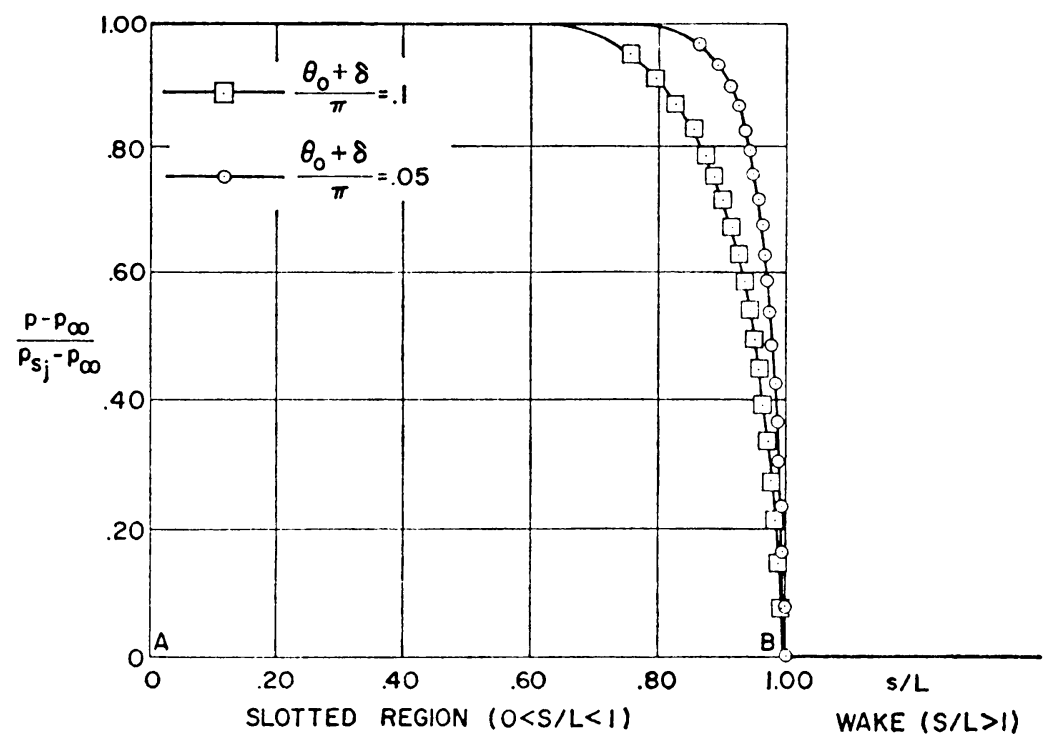

Fic. 6. Pressure distribution on the surface 
field of the injectant becomes a potential flow. For each model of flow field, with or without a separation region, the boundary conditions both upstream and downstream can be stated and included in the analysis and the theoretical solutions can be developed and examined.

The model of flow field without a separation region as shown in Fig. 2 can be ruled out on the following physical arguments. In order that injection on the lower surface will take place the inclination of the lower surface, $-\delta$, should not raise the pressure of the external stream to $p_{s i}$, i.e., $-\delta<\theta_{0}$. When $-\theta_{0}<\delta<\theta_{0}$, the inner flow field cannot form a concave corner with stagnation point at the trailing edge, $D$, because the pressure of the upper external flow at the trailing edge will be less than $p_{s i}$. On the other hand the inner flow field cannot form a convex corner at the trailing edge with pressure equal to $-\infty$ because it cannot match with the finite pressure of the outer flow field. The slope of the dividing streamline at the trailing edge has to be continuous. Consequently both the inclination and the pressure are specified at the trailing edge. Thus the boundary data are overspecified. When $\delta \geq \theta_{0}$, the inner flow field will have a concave corner at $D$. The pressure on the lower surface rises from $-\infty$ at the end $B$ of the slotted region to the stagnation value at the trailing edge $D$. For a real fluid, a separation bubble will be formed below the $90^{\circ}$ convex corner at $B$. The reattachment of the flow to the surface will not take place due to the adverse pressure gradicnt, i.e., there will be an infinite separation region.

In Sec. 2 an analytical solution for the potential flow field with a constant wake region is presented. The separation begins at the end of the injection region. Since it is an inviscid analysis and assumes a constant-pressure wake region, the solutions are independent of the distance between the injection region and the trailing edge. Of course, the distance should be such that the effects of the mixing along the dividing lines and the induced pressure gradient remain negligible until far downstream of the trailing edge. For the limiting case of the injection along a finite portion of an infinite wedge obtained in [7], it is assumed that there is no separation region. This is consistent with the classical inviscid analysis which ignores a small separation bubble next to the end of the injection region where the fluids turn around a $90^{\circ}$ convex corner. The solution with a constant-pressure wake in Sec. 2 and the solution for an infinite wedge without separation in [7] represent two limiting inviscid solutions. The solution to the real physical problem lies in between them. The viscous mixing along the dividing streamlines and the induced pressure gradient have to be included in the analysis to find out whether the wake is going to close in at the wing surface to form a closed bubble or to be diffused far downstream of the wing. Furthermore, when the speed of sound of the injectant is not too large, the compressibility effects have to be included and result in changes in flow patterns. For example, the inability of a potential flow to turn either a convex or a concave corner at the trailing edge will be removed if the flow of the injectant becomes transonic or supersonic near the trailing edge.

\section{REFERENCES}

11] J. Aros'y and J. D). Cole, Boumlary lityer flows with large injection rates, RA-4620-ARP.A, Aug. 5, The Rand Corp., Samt. Monica, California

[2] (i. R. Inger, An integral method solution for massive blowing on slender hypersonic bodies, AIAA J. 5, 2068-2069, (1967)

[3] J. D. Cole and J. Aroesty, The blowhard problem-inviscid flou's with surface injection, Internat. J. Heat, and Mass Trunsfer 11, 1167-1183 (1968) 
[4] J. Aroesty and S. H. Davis, Inviscid cone flows with surface mass transfer, AIAA J. 4, 1830-1832 (1966)

[5] G. Emanuel, Blowing from a porous cone or wedge when the contact surface is straight, AIAA J. 5, 534-538 (1967)

[6] J. Wallace and N. H. Kemp, Analytic solutions to the massive blowing problem, AIAA Preprint No. 68-128. Presented at AIAA 6th Aerospace Science Meeting, Jan. 1968

[7] L. Ting, Pressure distribution on a surface with large normal injection, AIAA J. 4, 1573-1579 (1966)

[8] A. Ismail, Large rate of injection normal to surface in high speed flow, Ph.D. thesis, School of Engineering and Science, N.Y.U., 1967

[9] K. Stewartson, On asymptotic expansions in the theory of boundary layers, J. Math. Phys. 36, 173191 (1957)

[10] L. Ting and S. Chen, Perturbation solutions and asymptotic solutions in boundary layer theory, J. Engrg. Math. 1, 327-340, (1967)

[11] H. Schlichting, Boundary layer theory, McGraw-Hill, New York, 1955

[12] A. Ferri, Elements of aerodynamics of supersonic flows, Macmillan, New York, 1949

[13] W. D. Hayes and R. F. Probstein, Hypersonic flow theory, Academic Press, New York, 1958, pp. 253-283

[14] L. Ting and A. Ismail, Large rate of normal injection near trailing edge, NYU-AA-68-21, 1968, New York Univ.

[15] L. M. Milne-Thomson, Theoretical hydrodynamics, 3rd ed., Macmillan, New York, 1952, pp. 279-297

[16] E. C. Titchmarsh, The theory of functions, 2nd ed., Oxford Univ. Press, New York, 1939 